\title{
OPTIMIZING BRAKING ENERGY FLOW THROUGH CHARGING STATUS SURFACE EXPANSION
}

\author{
Ivan Župan ${ }^{1 *}$ - Marko Lelas ${ }^{2}$ - Željko Ban ${ }^{3}-$ Viktor Šunde $^{4}$
}

\begin{abstract}
${ }^{1}$ Department of Control and Computer Engineering, Faculty of Electrical Engineering and Computing, University of Zagreb, Unska 3, 10000 Zagreb

${ }^{2}$ Department of Control and Computer Engineering, Faculty of Electrical Engineering and Computing, University of Zagreb, Unska 3, 10000 Zagreb

${ }^{3}$ Department of Control and Computer Engineering, Faculty of Electrical Engineering and Computing, University of Zagreb, Unska 3, 10000 Zagreb

${ }^{4}$ Department of Electric Machines, Drives and Automation, Faculty of Electrical Engineering and Computing, University of Zagreb, Unska 3, 10000 Zagreb
\end{abstract}

\begin{tabular}{|c|c|}
\hline ARTICLE INFO & Abstract: \\
\hline $\begin{array}{l}\text { Article history: } \\
\text { Received: } 23.12 .2019 . \\
\text { Received in revised form: } 10.1 .2020 . \\
\text { Accepted: } 14.1 .2020 . \\
\text { Keywords: } \\
\text { Braking energy } \\
\text { Optimization } \\
\text { Supercapacitor } \\
\text { energy flow control } \\
\text { power grid stability } \\
\text { control surface } \\
\text { DOI: https://doi.org/10.30765/er.1567 }\end{array}$ & $\begin{array}{l}\text { Energy savings in electric railway transportation is essential due } \\
\text { to the ever-rising energy cost and endeavour to reduce climate } \\
\text { change impact. A valuable method to increase energy efficiency } \\
\text { is to recuperate and consequently utilize the regenerative braking } \\
\text { energy of electric railway vehicles. The system that stores and } \\
\text { reuses the braking energy is called a regenerative braking } \\
\text { system, consisting of an energy storage system (ESS), a } \\
\text { bidirectional power converter, and a control system, which } \\
\text { includes an algorithm controlling the braking energy flow. A } \\
\text { properly designed algorithm increases energy efficiency, lessens } \\
\text { the stress on the power grid, increases the lifetime of the energy } \\
\text { storage system, and enables a catenary-free operation of the } \\
\text { electric railway vehicle. The algorithm is defined by combining } \\
\text { two algorithms with opposite features - maximum energy savings } \\
\text { and a minimal number of cycles. The algorithm is then } \\
\text { synthesized from those two criteria using an optimization process } \\
\text { and then simulated while its effect on energy savings and grid } \\
\text { stability is analyzed. Energy savings and a more stable grid are } \\
\text { achieved with the use of the algorithm, which corroborates the } \\
\text { inclusion of a regenerative braking system in electric railway } \\
\text { vehicles. }\end{array}$ \\
\hline
\end{tabular}

\section{Introduction}

Electric railway transportation is a widespread type of public transportation, which is characterized by its low price, high safety and low carbon footprint. The trend of increasing energy savings dictates an increase in energy efficiency in this type of transportation. One way to increase energy efficiency is to use the regenerative braking energy of railway vehicles. During braking, the electric railway vehicle's main drive acts like a generator in which the kinetic energy of the vehicle converts to electric energy. The generated electric energy is generally sent back to the power grid if the grid can accept it, or dissipated on the brake resistors.

A more efficient approach consists of storing the braking energy into energy storage systems (ESS) and to utilize it afterwards. Besides saving energy, the system for braking energy storage and subsequent usage (also called regenerative braking system) also increases grid voltage stability and enables autonomous, catenary-free driving. The stored energy is used during acceleration, which lowers the stress on the grid,

\footnotetext{
* Corresponding author

E-mail address: ivan.zupan@fer.hr
} 
helping stabilize its voltage. If the ESS is located inside the vehicle and is well dimensioned, a limited catenary-free operation is enabled. The basic components of a regenerative braking system are the energy storage system, the power converter to condition the braking energy and power flow, and the control system which includes the algorithm for charging and discharging the energy storage system.

The energy storage system can be located inside the vehicle (on-board storage system) or outside the vehicle in energy substations (wayside storage system). Storing the energy on-board the vehicle results in energy savings of up to 40\% [1-6]. The energy storage system generally consists of supercapacitor or lithium-ion cells with nominal voltage values typically between 2.5 and $3 \mathrm{~V}$. To achieve the necessary values of power and energy capacity, the cells are connected in series and parallel into modules. Within the energy storage system is also the battery management system (BMS) which has multiple functions $[7,8]$. Its function is to balance the voltage levels on specific cells, monitoring the state of charge and diagnose the state of health of the module.

The power converter that connects the main drive's DC link capacitor and the ESS is a bidirectional DC/DC converter [9, 10]. The converter's topology depends on the criteria set by the electric railway vehicle and the ESS. The algorithm within the converter's control system controls the energy flow through the bidirectional DC/DC converter. By carefully choosing the algorithm, higher energy efficiency can be achieved, as well as a longer ESS lifetime.

The braking energy flow algorithms described in $[11,12]$ are used in conjunction with wayside energy storage systems to stabilize the grid voltage. In [13, 14], the algorithms are more complex, but used with onboard energy storage systems. In [13], the non-linear braking energy model is separated into a group of linear subsystems, but the energy efficiency of the system is not emphasized. The algorithm presented in [14] describes a relatively simple algorithm which strives to conserve the supercapacitor module state of charge at a certain level, but it also sacrifices the possibility for higher energy efficiency.

In this paper, a braking energy flow algorithm is presented whose purpose is to achieve maximal braking energy storage alongside a minimal number of cycles of the supercapacitor module. The development of the railway vehicle, supercapacitor module and power grid models using an optimization procedure are described in chapter 2. Chapter 3 describes the development of the presented algorithm based on the two contrasting criteria. Chapter 4 shows the results of the algorithm implementation using the developed models and the measured operating tramway velocity profile.

\section{Railway vehicle, power grid, and supercapacitor modelling}

To develop the algorithm for the charge and discharge of the supercapacitor module, a vehicle model is constructed alongside a power grid model and an ESS model. The primary requirement of the model is an accurate enough description of the grid current and grid voltage behaviour in correlation with the tramway velocity and acceleration in order to accurately represent the flow of braking energy. The model must also be simple enough so that the optimizing procedure of the braking energy flow control algorithm can be conducted. The block scheme of the complete system is shown in Figure 1.

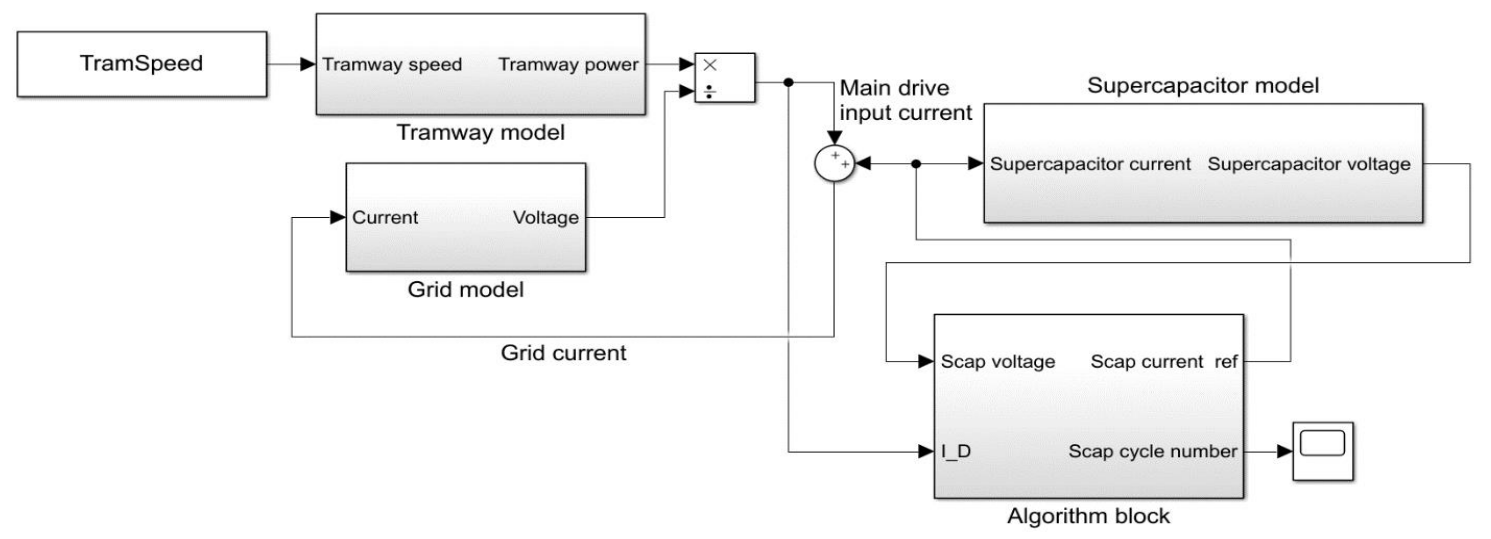

Figure 1. Block scheme of the tramway, grid, supercapacitor and algorithm models. 
The tramway model is defined by the physical laws of motion of vehicles and electrical phenomena in the grid according to power requirements during accelerating and braking. For the given (measured) tramway speed profile, the model outputs the main drive converter input current (i.e. the tramway input current). The grid current is the sum of the tramway input current and the supercapacitor module current, whose value and flow direction is determined by the algorithm block.

The system model parameters are defined using measurements from a tramway TMK 2200_K (manufactured by Končar Elektroindustrija Zagreb and TŽV Gredelj Ltd. ), without an integrated ESS [15]. The measurements were conducted with a Kvaser Memorator HS/SWC device connected through a CAN open bus that can record measurements on an SD card with a sampling time of $50 \mathrm{~ms}$. The list of recorded signals is shown in Table 1. Figure 2 shows a segment of the measured input current and the tramway speed during a typical acceleration and deceleration period.

Table 1. - Measured values during tramway operation on the line 11 Črnomerec - Dubec-Črnomerec.

\begin{tabular}{|l|c|}
\hline \multicolumn{1}{|c|}{ Value } & Unit \\
\hline $\begin{array}{l}\text { Main drive converter 1,2 and 3 input } \\
\text { current }\end{array}$ & $\mathrm{A}$ \\
\hline $\begin{array}{l}\text { Estimated torque of motor pair 1,2 and } \\
3\end{array}$ & $\mathrm{Nm}$ \\
\hline $\begin{array}{l}\text { Main drive converter output current } \\
\text { RMS value }\end{array}$ & $\mathrm{A}$ \\
\hline Vehicle speed & $\mathrm{km} / \mathrm{h}$ \\
\hline Drive handle position & $\%$ \\
\hline $\begin{array}{l}\text { Mass of the first, second and third } \\
\text { tramway section }\end{array}$ & $\mathrm{kg}$ \\
\hline
\end{tabular}

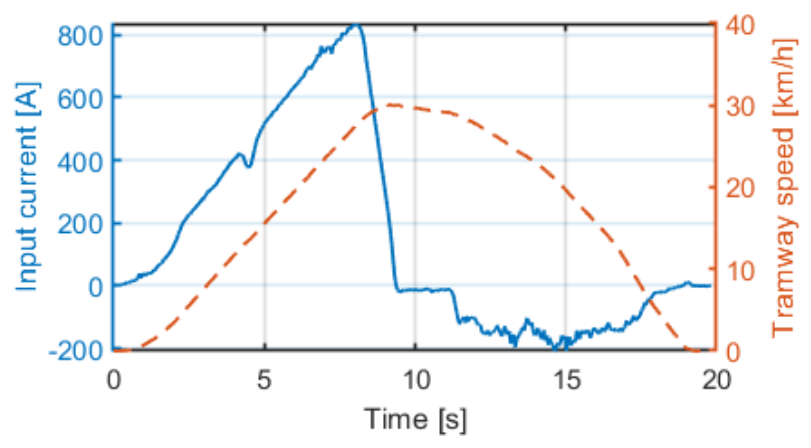

Figure 2. Waveform of measured input current and tramway speed during acceleration and deceleration.

\subsection{Power grid modelling}

Considering that the catenary voltage measurement is not available, the catenary voltage is determined using a simple power grid model alongside the measured input current. The grid model consists of an ideal DC voltage source in series with an RL circuit, Figure 3.

The model does not take into account the influence of other tramways on the same catenary, as well as the distance of the tramway from its power substation. The parameters of the grid model are given in Table 2. [15]

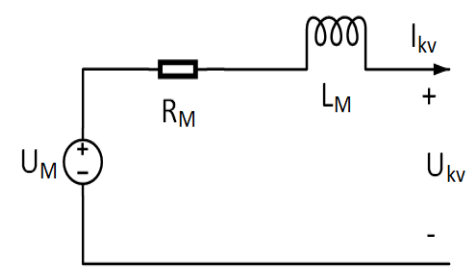

Figure 3. Power grid model representation. 
Table 2. Grid model parameters.

\begin{tabular}{|c|c|}
\hline Parameter & Value \\
\hline $\mathrm{U}_{\mathrm{m}} / V$ & 600 \\
\hline $\mathrm{R}_{\mathrm{m}} / \Omega$ & 0.0387 \\
\hline $\mathrm{L}_{\mathrm{m}} / H$ & 0.0023 \\
\hline
\end{tabular}

Figure 4 shows the output of the power grid model, i.e. the catenary voltage for the given tramway input current noticeable in Figure 2.

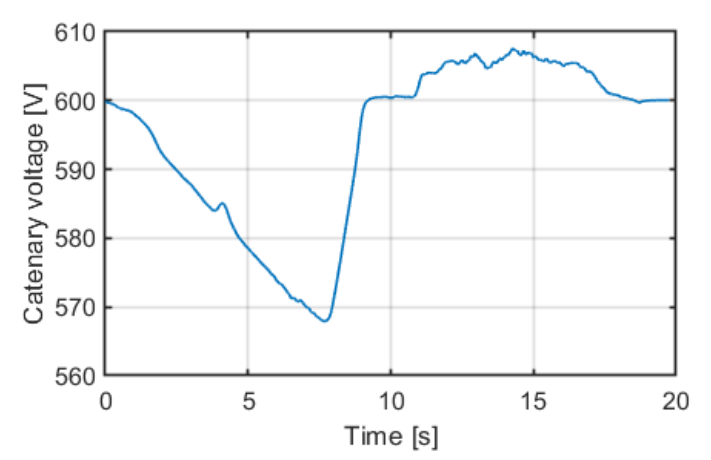

Figure 4. Simulated catenary voltage waveform.

\subsection{Railway vehicle mathematical model}

The tramway is modelled as a system of mass $m$ whose braking and accelerating power is determined from its instantaneous speed $v$ and acceleration $a$. Assuming that the tramway is driving on a straight line, with a slope angle $\alpha$, the total force acting on the tramway is described by equation (1):

$$
f_{t}=f_{i}+f_{v}+f_{r r}+f_{g}+f_{b}
$$

where $f_{i}$ is the inertial force, $f_{v}$ is the viscous damping force, $f_{r r}$ the rolling damping force, $f_{g}$ gravitational force and $f_{b}$ is the mechanical braking force. By identifying the aforementioned forces, a precise mathematical model of the vehicle can be developed. Due to the complicated process of determining the parameters of each force and the inability to conduct the necessary measurements, this paper uses the Davis formula to model the tramway. This formula is used to calculate the resistive force of the vehicle $f_{r}$ which substitutes the viscous damping force $f_{v}$ and the rolling damping force $f_{r r}$ [16]. The basic form of the formula is as follows:

$$
f_{r}=A v^{2}+B v+C
$$

where the coefficients A, B and C are calculated from the known physical characteristics of the vehicle and driving conditions (such as going through a tunnel). Throughout the literature, many methods for calculating the coefficients are given, but in this paper, an optimizing method was used to determine the coefficient values.

The tramway speed profile upon which the measurements were conducted does not have any significant change of altitude so the influence of the gravitational force can be ignored. By also ignoring the mechanical braking force, equation (1) becomes:

$$
f_{t}=f_{i}+f_{r}=m \frac{d v}{d t}+A v^{2}+B v+C
$$

By knowing the force $f_{\mathrm{t}}$ and the tramway speed, the power used for driving the tramway is:

$$
p_{t}=f_{t} \cdot v
$$




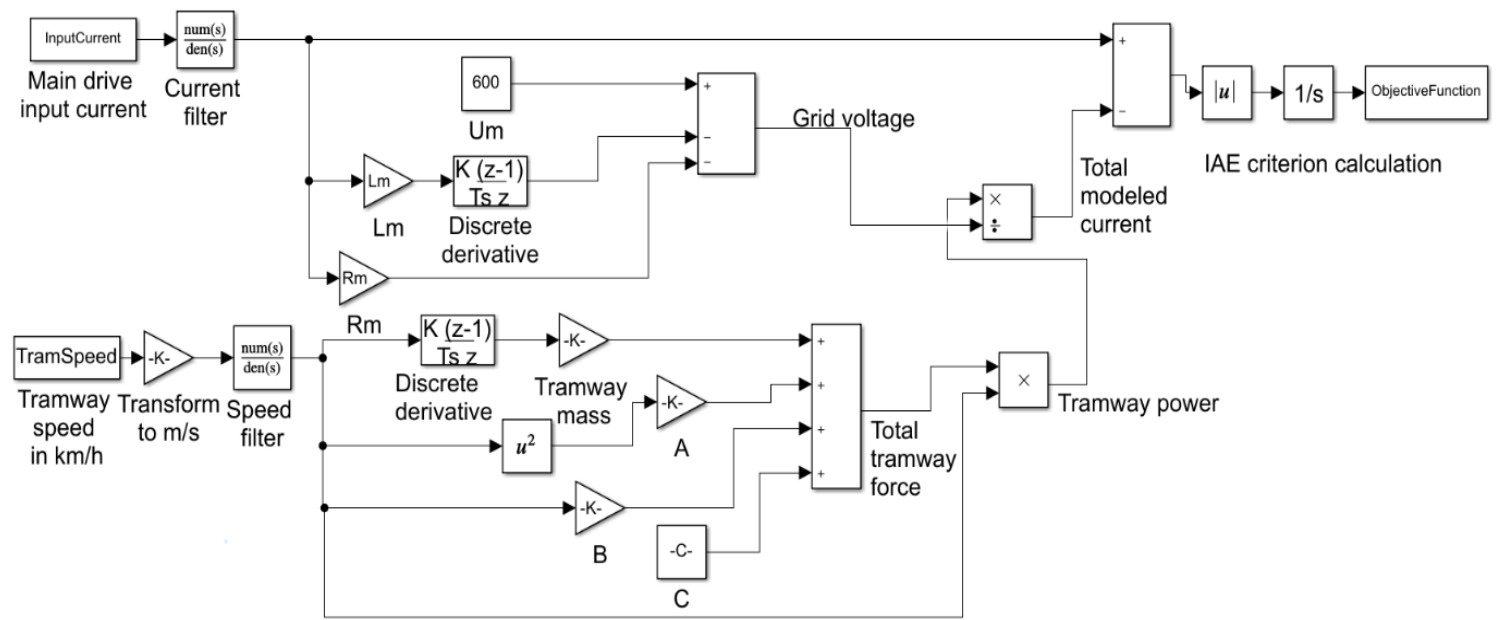

Figure 5. Simulation model of the tramway and power grid in MATLAB/Simulink with an optimization procedure for tunning $A, B$ and $C$.

The driving torque is given in equation (5):

$$
M_{t}=f_{t} \cdot r_{w}=\mu_{G} \cdot i \cdot M_{S}
$$

where $r_{w}$ is the tramway wheel radius. $M_{S}$ is the torque on the motor shaft, $i$ is the gear ratio between the motor shaft and the wheel shaft with a transmission efficiency $\mu_{G}$. The angular velocity of the motor shaft $w_{s}$ and the wheel $w_{w}$ is shown in the following equation:

$$
w_{s}=i \cdot w_{W}
$$

The motor's input power $p_{m}$ can be defined as the ratio of the mechanical power of the motor shaft $p_{s}$ and the motor efficiency $\mu_{m}$ :

$$
p_{m}=\frac{p_{s}}{\mu_{m}}=\frac{M_{S} w_{S}}{\mu_{m}}
$$

Combining the previous equations gives the following equation for the input power of the motor:

$$
p_{m}=\frac{f_{t} v}{\mu_{m} \mu_{G}}
$$

The motors are controlled by a variable-frequency drive with an efficiency $\mu_{f c}$. The variable-frequency drive input power $p_{d}$ is:

$$
p_{d}=\frac{p_{m}}{\mu_{f c}}=\frac{f_{t} v}{\mu_{f c} \mu_{m} \mu_{G}}
$$

In order to simplify the mathematical model, instead of the six real electric motors used to drive the tramway, an equivalent one is used. Using the law of conservation of power, the power taken from the grid $p_{\mathrm{kv}}$ must be equal to the sum of the vehicle power (variable-frequency drive) $p_{d}$, the power dissipated on braking resistors $p_{b}$ and the auxiliary power $p_{a}$ :

$$
p_{k v}=p_{d}+p_{b}+p_{a}
$$

With that, the modelled converter current $i_{d}^{\text {mod }}$ can be calculated by equation (11):

$$
p_{d}=p_{k v}-p_{b}-p_{a}=U_{k v}\left(i_{k v}-i_{b}-i_{a}\right)
$$




$$
\begin{aligned}
& p_{d}=\frac{f_{t} v}{\mu_{f c} \mu_{m} \mu_{G}}=U_{k v}\left(i_{k v}-i_{b}-i_{a}\right) \\
& i_{d}^{\text {mod }}=i_{k v}=\frac{f_{t} v}{U_{k v} \mu_{f c} \mu_{m} \mu_{G}}+i_{b}+i_{a}
\end{aligned}
$$

In the railway vehicle model (11), losses are ignored, i.e. it is assumed that $\mu_{f c}, \mu_{M}$ and $\mu_{G}$ are equal to 1 . It is also assumed that the currents $i_{\mathrm{a}}$ and $i_{\mathrm{b}}$ are equal to zero (because there are no measurements made for them). To use the simplified equation (11), besides the measured speed $v$, it is necessary to know the values of the grid voltage $U_{\mathrm{kv}}$ and the force $f_{\mathrm{t}}$. The parameters $A, B$ and $C$ from the Davis equation (2) are initialized through an optimization procedure using the IAE (Integral Absolute Error) criterion and the tramway model within the Simulink in MATLAB, shown in Figure 5. The tramway input current and speed measurements, recorded during cca 21 operating hours, are used as an input for the optimization procedure.

To speed up the optimizing procedure, a shorter sample from the measurements is used as input, with a length of $19.8 \mathrm{~s}$. The measurements are filtered to avoid the influence of input signal discrete nature on the derivatives in the model. The used filter is a simple PT1 filter with a time constant of $0.15 \mathrm{~s}$, which is three times the sample time. The optimizing results are presented in Table 3.

Table 3. Tramway model parameters.

\begin{tabular}{|l|l|}
\hline Parameter & Value \\
\hline Tramway mass / kg & 49373 \\
\hline $\mathrm{A}$ & 17.965 \\
\hline $\mathrm{B}$ & 34.536 \\
\hline $\mathrm{C}$ & 7827.25 \\
\hline
\end{tabular}

Using the calculated parameters, the tramway model connected to the grid model in Figure 5, and with parameters from table 2, is using the measured input current shown in Figure 6. From Figure 6, the modelled current reflects well the measured current during acceleration. However, during braking, the measured and modelled current differ in magnitude. The reason for this is the ignored parameters during the evaluation of the parameters of the power grid (the assumption that there are no other vehicles on the same catenary within the range of the same power substation) and ignoring the current in the braking resistor. Nevertheless, for the proposed algorithm development, the resulting input current is of satisfying accuracy.

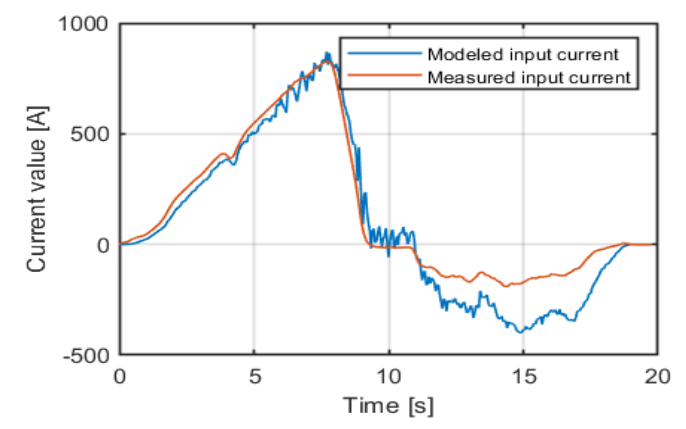

Figure 6. Comparison of modelled and measured tramway input current.

\subsection{Modelling the ESS}

The regenerative braking system typically consists of an energy storage system, a power converter and a control system which includes an algorithm for charging and discharging the energy storage system. The regenerative braking system's quality depends heavily on the quality of the used algorithm that controls the energy flow between the electric railway vehicle and the energy storage system.

The power converter is a bidirectional DC-DC converter modelled as an ideal converter whose current output can be controlled. Since its dynamic is negligible compared to the dynamic of the rest of the system, its transfer function is not taken into account for the system modelling. 
The energy storage system, i.e. the module constructed from supercapacitor cells, is modelled as a series $\mathrm{RC}$ circuit consisting of a capacitance $C_{s c}$ and resistance $R_{s c}$, Figure 7.

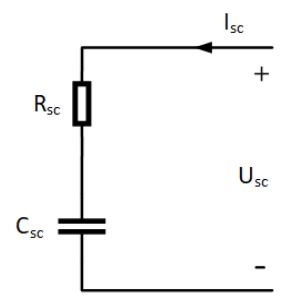

Figure 7. Supercapacitor module model representation.

Per Figure 7, the following equation represents the supercapacitor voltage:

$$
U_{s c}=i_{s c} R_{s c}+\frac{1}{c_{s c}} \int i_{s c} d t
$$

The supercapacitor equivalent model shown on Figure 7, represents the series connection of four supercapacitor modules Maxwell BMOD0063 P125 B08. From the manufacturer catalogue, the nominal voltage value of the module series is $500 \mathrm{~V}$. Per manufacturer recommendation, the module should not be discharged below $250 \mathrm{~V}$ and charged or discharged, with a current magnitude above $240 \mathrm{~A}$.

\section{Energy flow control algorithms}

While considering an algorithm for braking energy flow control, a starting point is to take two basic algorithms. The first algorithm is based on controlling the energy flow while satisfying the constraint to prolong the lifetime of the supercapacitor module by minimizing the number of cycles. A concrete definition of a cycle cannot be found throughout the literature. In this paper, a cycle is considered as a change of energy flow direction in the supercapacitor module. The operating principle of this energy flow control algorithm is based upon charging the supercapacitor until it is fully charged, or discharging the supercapacitor until it is discharged down to $50 \%$ of its nominal voltage value, after which the energy flow is changed from charging to discharging and vice versa. Aside from a prolonged supercapacitor module lifetime, the negative consequence of this algorithm is a decreased energy efficiency, e.g. during the discharging phase, the ESS cannot be charged until it is completely discharged.

The second algorithm is based on the idea to recuperate a maximum amount of energy during braking instead of dissipating it on brake resistors and to use the maximum amount of energy during acceleration. While this approach features higher energy efficiency, it can also be beneficial to stabilize the grid voltage. Instead of returning the energy to the grid, when other tramways cannot accept it, it can be stored in the ESS. The downside of this algorithm is that it severely reduces the lifetime of the ESS because it accepts the braking energy from every braking situation, and delivers energy during every acceleration. In addition, if the ESS is poorly designed, a part of the braking energy can still be dissipated on braking resistors or sent back to the grid causing instability.

The algorithm proposed in this paper contains in itself the advantages of both aforementioned algorithms. The goal is to store the maximum amount of energy during the charging status and to satisfy the energy demands during the discharging status while using a minimum number of cycles. The change of charging/discharging status does not happen when the ESS is fully charged or discharged; the change in status occurs depending on the energy demand of the vehicle and state of charge. To analyse the behaviour of the proposed algorithm, it is implemented in the block scheme of the system shown in Figure 1, inside the block named Algorithm block. The algorithm determines whether the supercapacitor releases energy during acceleration, or accepts energy during braking. The simulation is carried on a speed profile representative of a tramway line which is about 2500 seconds long, shown in Figure 8. 


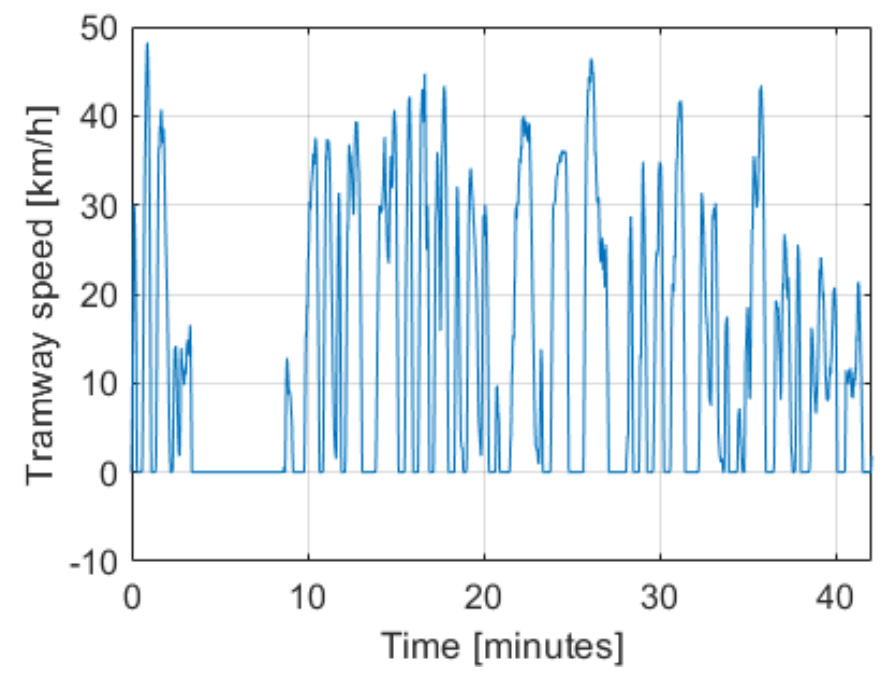

Figure 8. Measured speed profile of tramway driving.

Figure 9 shows the projection of control surfaces for charging and discharging statuses, which depend on the state of charge of the ESS and the tramway input current.
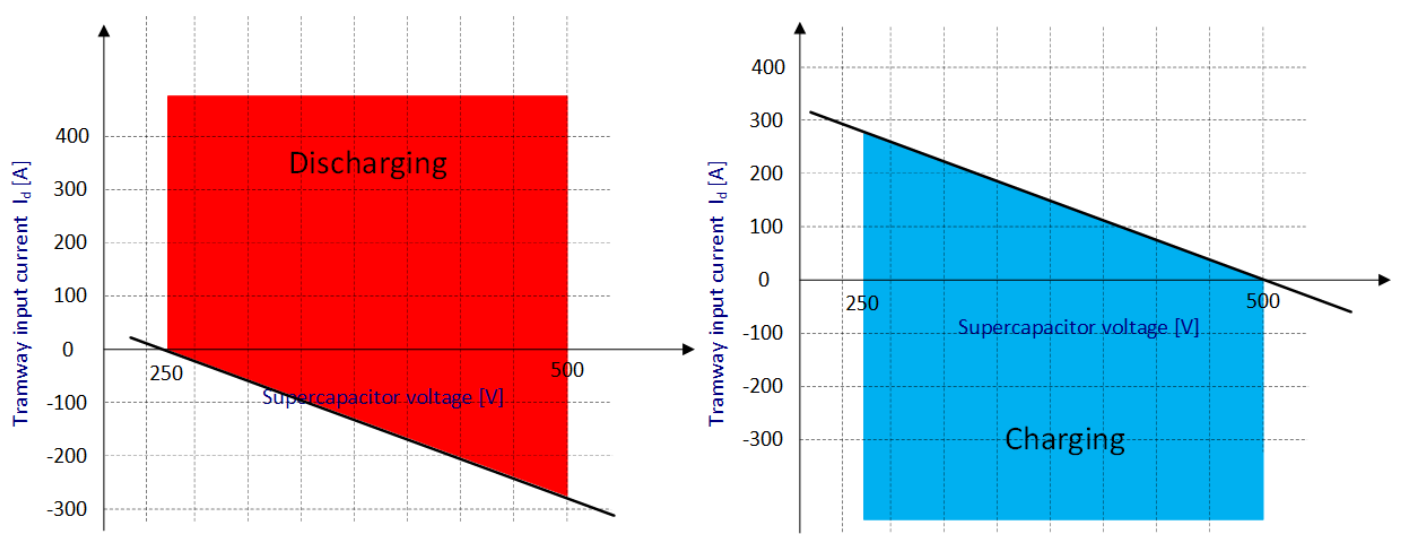

Figure 9. Projections of the control surfaces for the charging and discharging statuses.

The blue surface represents the ESS charging state, while the sloped line represents the transition boundary from the charging to discharging state. For the ESS in charging state, all negative values of the tramway input current, i.e. during braking, are directed to ESS. This algorithm allows staying in the charging status even during acceleration, i.e. for positive values of the tramway input current up to the boundary line. It means that for charging status, ESS energy is not used for acceleration until the boundary line is crossed. However, for positive current values that are not within the charging surface (above boundary line), the ESS state changes from charging to discharging. It can be seen that small values of tramway input current, near the maximum allowed voltage value of $500 \mathrm{~V}$, cause a change from charging status to discharging status, compelling the discharge of the (near) fully charged supercapacitor. The red surface represents the discharging state of ESS. For the ESS in discharging state, the system remains in the same state for all positive currents and even for negative values of the tramway input current which are not below the boundary line.

Negative current values (i.e. braking current) below the boundary line, change the status to charging. It can also be seen that small braking current values near the minimal voltage $(250 \mathrm{~V})$ induce a change in status from discharging to charging. The algorithm operation of charging is described with the flow diagram in Figure 10. 


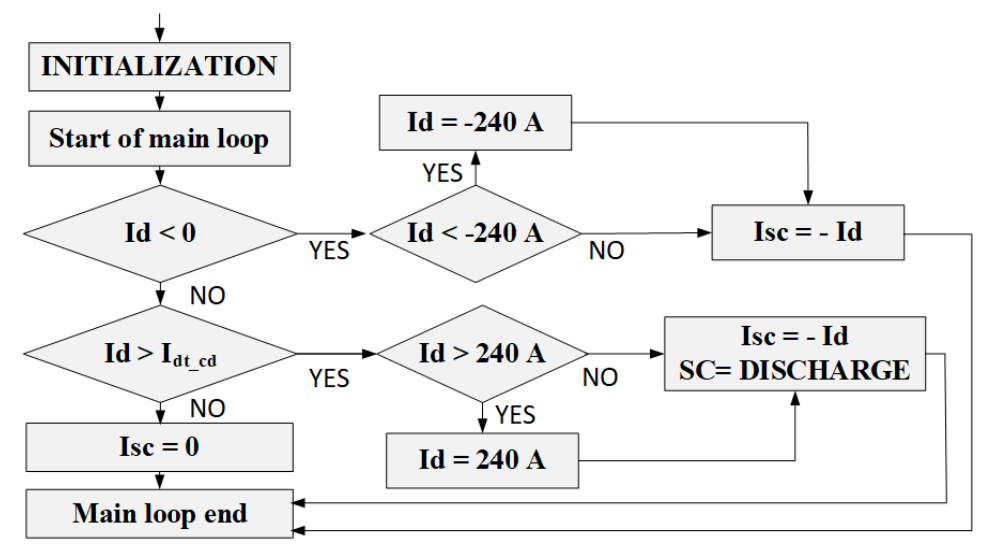

Figure 10. Flow diagram of the algorithm operation during charging status.

The Figure 10 shows the algorithm operation during charging status in which the decision is made about changing the status, as well as stopping the charging if the voltage value is equal to or higher than $500 \mathrm{~V}$, or discharging if the voltage value is lower than or equal to $250 \mathrm{~V}$ which is set during the initialization of the algorithm. The lines that determine the status surfaces are defined in the following equations:

$$
I_{d t_{-} c d}=-I_{c d}\left(U_{S C}-U_{S C \operatorname{Max}}\right)
$$

where:

$I_{d t \_d}-$ the boundary line of status change from charging to discharging $I_{d t \_d c}-$ the boundary line of status change from discharging to charging $I_{c d}$ - the slope of the line of status change from charging to discharging $I_{d c}$ - the slope of the line of status change from discharging to charging $U_{s c}$ - instantaneous voltage value of the supercapacitor module $U_{\text {sc Min }}$ - minimal allowed voltage value of the supercapacitor module $U_{s c}$ Max - maximal allowed voltage value of the supercapacitor module

Determining the slope coefficients $I_{c d}$ and $I_{d c}$ is conducted with an optimization algorithm. Practically, there are two methods to determine the optimal values of the slope coefficients $\mathrm{I}_{\mathrm{cd}}$ and $\mathrm{I}_{\mathrm{dc}}$. For both methods, it is necessary to define the objective function as well as additional conditions and constraints. In this particular case, the objective function can be defined by two approaches: the ratio of the total stored energy and the number of cycles or the reciprocal value of that ratio. For the first ratio, the maximum possible value is pursued while for the second, its minimal value. In order to use MATLAB optimization tools for obtaining minimums, it is suitable to choose the ratio of the number of cycles and the total stored energy as a criterion function.

The first method to determine the optimal coefficient values $I_{c d}$ and $I_{d c}$ is of heuristic nature. It is based on using a MATLAB script to simulate the model shown in Figure 1 with different combinations of $I_{c d}$ and $I_{d c}$. This method is both demanding on the computer processor and time-consuming, but it can give a good illustration of the coefficient values $I_{c d}$ and $I_{d c}$. A graphical representation of this method is shown in Figure 12, and Figure 13 shows an enhanced plot near the minimum value.

The second method for determining the optimal values of coefficients $I_{c d}$ and $I_{d c}$ is based on using the fminsearch command within MATLAB to find the values of $I_{c d}$ and $I_{d c}$ that give the minimum value of the objective function. The command calls another $\mathrm{m}$-function that simulates the model given in Figure 1 with the coefficients $I_{c d}$ and $I_{d c}$ which are different in each iteration until the minimum value of the objective function is found. The plot representing the progression of the objective function value is shown in Figure 14. 


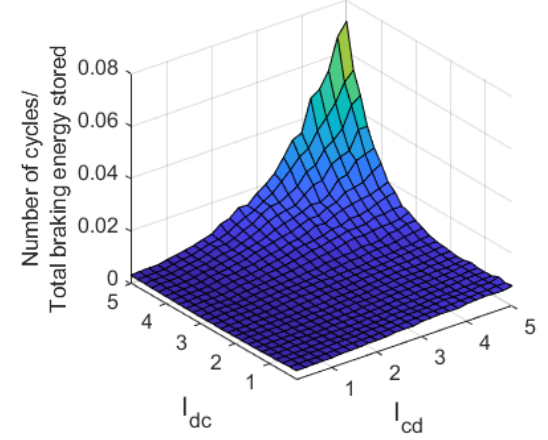

Figure 12. Objective function $3 D$ plot for different values of $I_{c d}$ and $I_{d c}$.

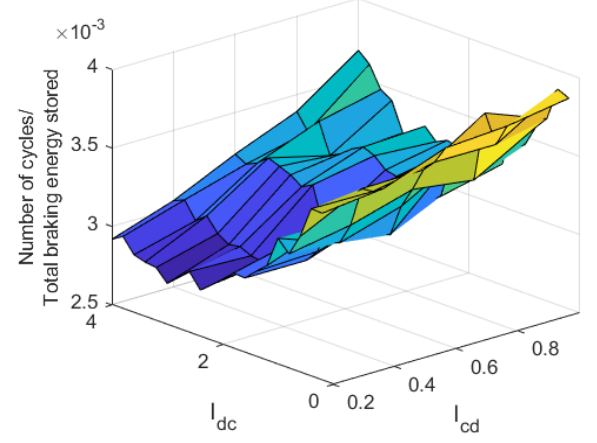

Figure 13. Objective function $3 D$ plot for different values of $I_{c d}$ and $I_{d c}$ enhanced near the minimum.

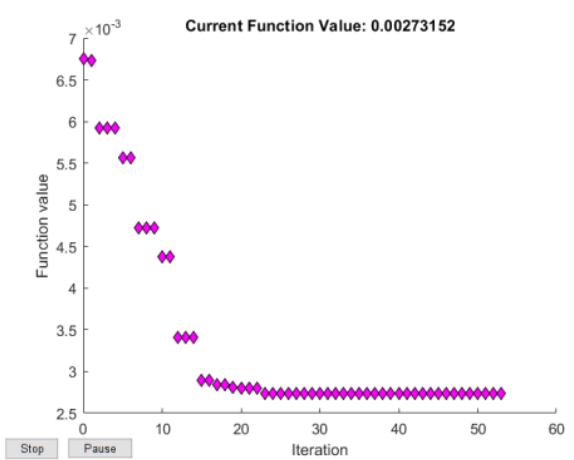

Figure 14. Objective function value progression plot in MATLAB.

The final result of the optimization process are the coefficient values $I_{c d}=0.17375$ and $I_{d c}=3.01875$. Using these coefficients give 79 cycles and a total stored energy value of $28.88 \mathrm{kWh}$. Compared with the algorithms discussed at the beginning of this section, this algorithm uses 3.43 times more cycles, but stores 1.95 times more energy in comparison with the algorithm whose main goal is to prolong the supercapacitor lifetime. In relation to the maximum stored energy algorithm, it uses 1.73 times fewer cycles, but stores 1.18 times less energy.

It is also worth showing the effect the proposed algorithm has on the grid current and voltage.

The grid current waveform with and without the implemented algorithm is shown in Figure 15. The Figure 16 shows the stabilizing effect caused by the algorithm on the grid voltage. It can be seen that the grid voltage is more stable with the implementation of the proposed algorithm, which lowers the possibility of grid overvoltage, and undervoltage, during operating times. 

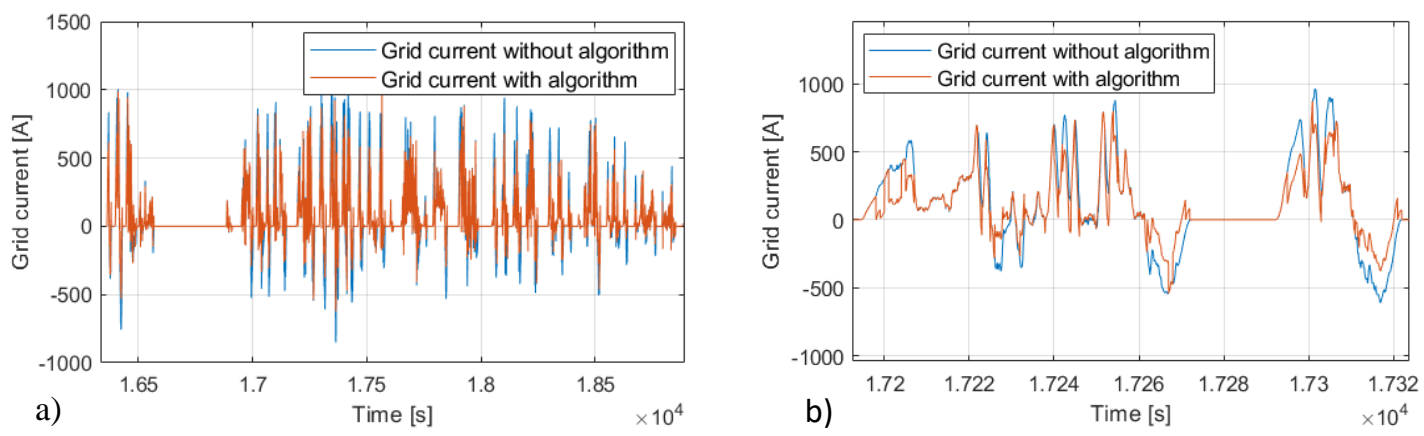

Figure 15. a) Comparison of grid current waveforms with and without algorithm operation b) During a smaller period.
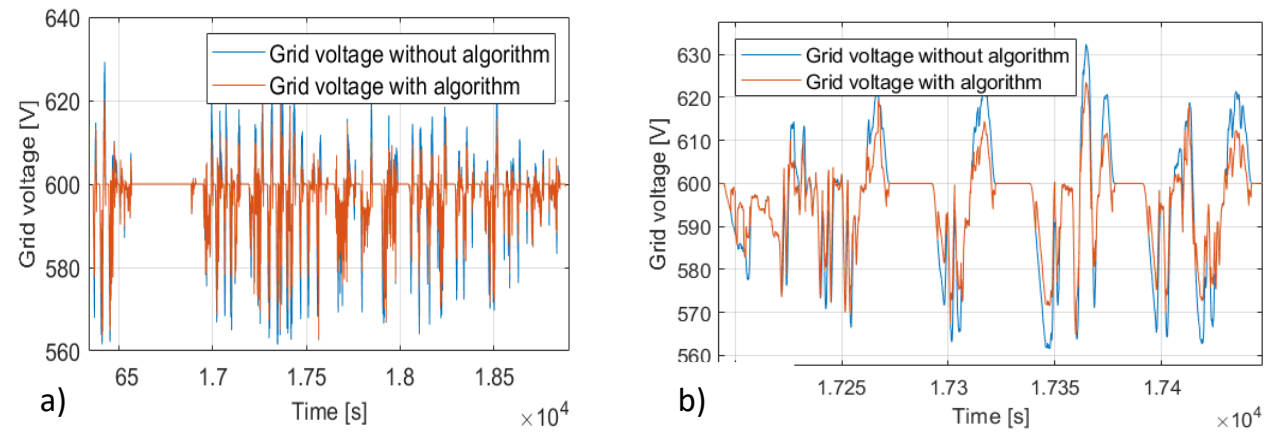

Figure 16. a) Comparison of grid voltage waveforms with and without algorithm operation b) During a smaller period.

\section{Conclusion}

The use of a regenerative braking system provides an efficient solution for storing the electric railway vehicle's braking energy and subsequent use of the stored energy. This way, energy savings are achieved, the impact on the power grid is decreased and possibly, a catenary-free operation is enabled.

This paper presents and develops an energy flow control algorithm between the DC link capacitor of the vehicle and the supercapacitor module, which is established upon two essential conditions; maximizing the stored energy and minimizing the number of supercapacitor cycles in order to accumulate and use as much energy as possible, while in the same time extending the supercapacitor lifetime.

To develop the energy flow control algorithm, a model of the complete system has been created. The power grid, supercapacitor module, and tramway models were created using measurements from real operating conditions. The tramway model has been developed using an optimization process within the MATLAB programming environment.

The developed algorithm is realized by extending the control surfaces of the maximum stored energy algorithm and the minimum cycle number algorithm. The extension is realized by boundary lines which define the transition from charging to discharging state and vice versa. The line slopes are determined using the optimization tool within MATLAB.

An indicative result of the proposed algorithm using the measured speed profile is that using 79 cycles, $28.88 \mathrm{kWh}$ of energy is stored inside the energy storage system. In comparison with the minimum cycle algorithm, this is 3.43 times more cycles, but 1.95 times more energy stored; compared to the maximum stored energy algorithm, it is 1.73 times fewer cycles, but 1.18 times less energy stored. The simulation results also indicate that the grid voltage is more stable after the implementation of the energy storage system and the proposed algorithm, which lowers the chance of power grid failures during operating conditions.

The subsequent research would be to determine whether using a higher-order polynomial instead of a line would save more energy, and use fewer cycles within this algorithm. 


\section{References}

[1] Gonzalez-Gil, A., Palacin R., Batty P., Sustainable urban rail systems: Strategies and technologies for optimal management of regenerative braking energy, Energy Conversion and Management, Volume 75(November 2013), 374-388

[2] Ogasa, M., 2010, Application of Energy Storage Technologies for Electric Railway VehiclesExamples with Hybrid Electric Railway Vehicles, IEEJ Transactions on Electrical and Electronic Engineering, 2010, 5: 304-311

[3] Del Pizzo, A., Fratelli L., Perna D., Nappo C., A method for "design to range” for energy storage systems in catenary free operations of light railway vehicles, 2015 International Conference on Clean Electrical Power (ICCEP), Taormina, Italy, 2015

[4] Ratniyomchai T, Hillmansen S., Tricoli P., Optimal Capacity and Positioning of Stationary Supercapacitors for Light Rail Vehicle Systems, 2014 International Symposium on Power Electronics, Electrical Drives, Automation and Motion, Ischia, Italy, 2014

[5] Ciccarelli F., Iannuzzi D., Kondo K., Fratelli L., Line Voltage Control based on Wayside Energy Storage Systems for Tramway Networks, IEEE Transactions on Power Electronics, Volume 31(Jan. 2016), Issue $1,884-899$

[6] Ianuzzi D., E. Pagano E., Tricoli P., The Use of Energy Storage Systems for Supporting the Voltage Needs of Urban and Suburban Railway Contact Lines, Energies, 6(2013), Issue 4, 1802-1820

[7] Mohamed D., Noshin O., Van Den Bossche P., Van Mierlo J., Passive and Active Battery Balancing comparison based on MATLAB Simulation, 2011 IEEE Vehicle Power and Propulsion Conference, Chicago, IL, USA, 2011

[8] Navbir S., Patnaik L., Williamson S.S., Power Electronic Converters for Ultracapacitor Cell Balancing and Power Management: A Comprehensive Review, IECON - 42nd Annual Conference of the IEEE Industrial Electronics Society, Florence, Italy, 2016

[9] Stojadinovic M., Biela J., Comparison of High Power Non-Isolated Multilevel DC-DC Converters for Medium-Voltage Battery Storage Applications, 2015 17th European Conference on Power Electronics and Applications (EPE'15 ECCE-Europe), Geneva, Switzerland, 2015

[10] Park C.-H., Jang S.-J., Lee B.-K., Won C.-Y., Lee H.-M., Design and Control Algorithm Research of Active Regenerative Bidirectional DC/DC Converter used in Electric Railway, 2007 7th International Conference on Power Electronics, Daegu, South Korea, 2007

[11] Zhu F., Yang Z., Lin F., Xin Y., Dynamic Threshold Adjustment Strategy of Supercapacitor Energy Storage System based on No-load Voltage Identification in Urban Rail Transit, 2019 IEEE Transportation Electrification Conference and Expo, Asia-Pacific (ITEC Asia-Pacific), Seogwipo-Si, South Korea, 2019

[12] Xin Y., Yang Z., Lin F., Zhu F., Research on Energy Management Strategy of Supercapacitor Energy Storage System in Urban Rail Transit Based on Fuzzy Logic Control, 2019 IEEE Transportation Electrification Conference and Expo, Asia-Pacific (ITEC Asia-Pacific), Seogwipo-si, South Korea, 2019

[13] Xin Y., Yang Z., Lin F., Zhu F., Research on Energy Management Strategy of Supercapacitor Energy Storage System in Urban Rail Transit Based on Fuzzy Logic Control, 2019 IEEE Transportation Electrification Conference and Expo, Asia-Pacific (ITEC Asia-Pacific), Seogwipo-si, South Korea, 2019

[14] Xin Y., Yang Z., Lin F., Zhu F., Research on Energy Management Strategy of Supercapacitor Energy Storage System in Urban Rail Transit Based on Fuzzy Logic Control, 2019 IEEE Transportation Electrification Conference and Expo, Asia-Pacific (ITEC Asia-Pacific), Seogwipo-si, South Korea, 2019

[15] Xin Y., Yang Z., Lin F., Zhu F., Research on Energy Management Strategy of Supercapacitor Energy Storage System in Urban Rail Transit Based on Fuzzy Logic Control, 2019 IEEE Transportation Electrification Conference and Expo, Asia-Pacific (ITEC Asia-Pacific), Seogwipo-si, South Korea, 2019

[16] Sengor I., Kilickiran H.C., Akdemir H., Kilic B., Determination of Potential Regenerative Braking Energy in Railway Systems: A Case Study for Istanbul M1A Light Metro Line, 2016 5th International Conference on Power Science and Engineering, Venice, Italy, 2016 